\title{
Bulk Ingredient for Human Prescription Compounding
}

National Cancer Institute

\section{Source}

National Cancer Institute. Bulk Ingredient for Human Prescription Compounding. NCI Thesaurus. Code $C 96793$.

A category specifying that a bulk product is intended for use on humans and requires a prescription. 